\title{
Acute Effects of Frequent Light-Intensity Standing-Based Exercises That Interrupt 8 Hours of Prolonged Sitting on Postprandial Glucose in Stroke Survivors: A Dose-Escalation Trial
}

\author{
Paul Mackie, Gary Crowfoot, Prajwal Gyawali, Heidi Janssen, Elizabeth Holliday, \\ David Dunstan, and Coralie English
}

\begin{abstract}
Background: Interrupting prolonged sitting can attenuate postprandial glucose responses in overweight adults. The doseresponse effect in stroke survivors is unknown. The authors investigated the effects of interrupting 8 hours of prolonged sitting with increasingly frequent bouts of light-intensity standing-based exercises on the postprandial glucose response in stroke survivors. Methods: Within-participant, laboratory-based, dose-escalation trial. Participants completed three 8-hour conditions: prolonged sitting and 2 experimental conditions. Experimental conditions involved light-intensity standing-based exercises of increasing frequency $(2 \times 5 \mathrm{~min}$ to $6 \times 5 \mathrm{~min}$ bouts). Postprandial glucose is reported. Results: Twenty-nine stroke survivors (aged $66 \mathrm{y}$ ) participated. Interrupting 8 hours of prolonged sitting with light-intensity standing-based exercises every 90 minutes significantly decreased postprandial glucose (positive incremental area under the curve; $-1.1 \mathrm{mmol} / \mathrm{L} \cdot 7 \mathrm{~h} ; 95 \%$ confidence interval, -2.0 to -0.1$)$. In the morning (08:00-11:00), postprandial glucose decreased during the $4 \times 5$ minutes and $6 \times 5$ minutes conditions (positive incremental area under the curve; $-0.8 \mathrm{mmol} / \mathrm{L} \cdot 3 \mathrm{~h} ; 95 \%$ confidence interval, -1.3 to -0.3 and $-0.8 \mathrm{mmol} / \mathrm{L} \cdot 3 \mathrm{~h} ; 95 \%$ confidence interval, -1.5 to -0.2 , respectively) compared with prolonged sitting. Conclusion: Interrupting 8 hours of prolonged sitting at least every 90 minutes with light-intensity standing-based exercises attenuates postprandial glucose in stroke survivors. During the morning, postprandial glucose is attenuated when sitting is interrupted every 60 and 90 minutes.
\end{abstract}

Keywords: clinical research, exercise, metabolic health

Time spent in prolonged sitting is associated with detrimental impacts on cardiometabolic health, notably through alterations in lipid and glucose metabolism (dysglycemia). ${ }^{1-3}$ Dysglycemia is strongly associated with an increased risk of developing diabetes. ${ }^{4}$ Diabetes leads to an increased risk of mortality, reduced functional outcome, and increased risk of recurrent stroke in stroke survivors. $^{5-7}$ With a high prevalence of stroke survivors living with prediabetes (approximately $23 \%-53 \%)^{8,9}$ or diabetes (approximately $16 \%-43 \%),{ }^{5,9,10}$ improved glucose regulation is fundamental in the management of post stroke outcomes.

Mackie, Janssen, and English are with the School of Health Sciences and Priority Research Centre for Stroke and Brain Injury, The University of Newcastle, Newcastle, NSW, Australia. Mackie, Crowfoot, Janssen, and English are with the Centre for Research Excellence in Stroke Recovery and Rehabilitation, Florey Institute of Neuroscience and Mental Health, Melbourne, VIC, Australia. Crowfoot is also with the School of Nursing and Midwifery and the Priority Research Centre for Stroke and Brain Injury, The University of Newcastle, Newcastle, NSW, Australia. Gyawali is with the School of Biomedical Science and Pharmacy and the Priority Research Centre for Stroke and Brain Injury, The University of Newcastle, Newcastle, NSW, Australia; and the Hunter Medical Research Institute, Newcastle, NSW, Australia. Janssen is also with the Hunter Stroke Service, Hunter New England Local Health District, Newcastle, NSW, Australia. Holliday is with the School of Medicine and Public Health, University of Newcastle, Newcastle, NSW, Australia. Dunstan is with the Baker Heart and Diabetes Institute, Melbourne, VIC, Australia; and the Mary MacKillop Institute for Health Research, Australian Catholic University, Melbourne, VIC, Australia. Mackie (Paul.I.Mackie@uon.edu. $\mathrm{au}$ ) is corresponding author.
Regular engagement in physical activity is beneficial for managing dysglycemia and diabetes. ${ }^{11,12}$ Despite this, many stroke survivors retain sedentary lifestyles, ${ }^{13-15}$ spending up to $75 \%$ of their waking day sitting ${ }^{13,14}$ and this frequently coincides with low levels of physical activity (light intensity; 23\%, moderate- to vigorous-intensity physical activity; $0.5 \%) .{ }^{14}$ Promisingly, evidence suggests that interrupting prolonged sitting with brief bouts of physical activity or standing may attenuate postprandial glucose responses. ${ }^{2,16,17}$ Dempsey et al $^{18}$ found that among participants with type 2 diabetes, postprandial glucose was decreased by an average of $39 \%$ when 8 hours of prolonged sitting was interrupted with frequent short bouts (3 min every $30 \mathrm{~min}$ ) of resistance activities while standing (knee raises, calf raises, gluteal contractions, and mini squats). However, these findings are in contrast with that of an experimental trial completed in stroke survivors (BUSTStroke). ${ }^{19}$ In the BUST-Stroke trial, postprandial glucose was not attenuated when 8 hours of prolonged sitting was interrupted with 3-minute bouts of light-intensity standing exercises (calf raises, mini squats, and marching on the spot) or walking (every $30 \mathrm{~min}){ }^{19}$ These findings on the acute postprandial response in stroke survivors are unexpected, particularly since the BUST-Stroke trial reported significant benefits on other outcomes of cardiometabolic health (systolic blood pressure). ${ }^{20}$ Therefore, further investigation is warranted to characterize the effects of different doses of lightintensity standing exercises on the acute postprandial glucose response in stroke survivors.

We investigated the effects of interrupting 8 hours of prolonged sitting with different doses of light-intensity exercises while 
standing (STAND-EX) in stroke survivors. Specifically, the study aimed to examine the effect of increasing the frequency of lightintensity standing-based exercises on the postprandial glucose response.

\section{Methods}

\section{Design}

This manuscript reports results for the predetermined secondary outcome (postprandial plasma glucose) from a laboratory-based, dose-escalation trial, the methods of which have been previously described. ${ }^{21}$ Briefly, stroke survivors recruited from the Newcastle and Hunter region (NSW, Australia) attended the Hunter Medical Research Institute on 4 separate occasions (Figure 1). Participants undertook a familiarization session and three 8-hour conditions in cohorts of 10 participants. Conditions included a control condition (prolonged sitting) and 2 experimental conditions (sitting interrupted with light-intensity exercises [described below] while standing [STAND-EX]). Conditions increased in the frequency of STAND-EX bouts until a dose-limiting threshold was attained; inability of participants to achieve $80 \%$ of the target duration in STAND-EX and $\geq 70 \%$ of the cohort achieving the dose-limiting threshold. In brief and as detailed in Table 1, cohort 1 completed experimental condition $1(2 \times 5$ min STAND-EX bouts $)$ and experimental condition $2(4 \times 5 \mathrm{~min}$ STAND-EX bouts). Cohort 2 completed experimental condition $2(4 \times 5$ min STAND-EX bouts) and experimental condition $3(6 \times 5$ min STAND-EX bouts). At the dose-limiting threshold, we planned for a final cohort to complete the second to last cohort to confirm the optimal dose of STAND-EX. This trial was registered with the Australian New Zealand Clinical Trials Registry (ACTRN12617001517369) and approved by the University of Newcastle's Human Research Committee (H-2017-0296) and Hunter New England Research Ethics Committee (17/06/21/4.04). Prior to commencement, written and verbal informed consent was provided by all participants.

\section{Participants}

Adult stroke survivors ( $\geq 18 \mathrm{y} ;>3$ mo post stroke) were included if they: self-reported sitting for $\geq 7$ hours per day; and could walk at $\geq 0.4 \mathrm{~m} / \mathrm{s}$ with minimal assist (functional ambulation classification $\geq 3$ ). They were excluded if they: had a body mass index $>45 \mathrm{~kg} / \mathrm{m}^{2}$, had urinary frequency, self-reported moderate to vigorous physical activity $\geq 150$ minutes per week, took diabetes medication (other than metformin), were diagnosed with diabetes, were a smoker, or were pregnant.

The initial familiarization visit included collection of the following baseline characteristics: stroke profile (National Institute of Health Stroke Scale, ${ }^{22}$ Oxfordshire classification) ${ }^{23}$; walking speed (10-m walk test); type 2 diabetes mellitus risk (Australian type 2 diabetes risk assessment tool) ${ }^{24}$; fatigue (Fatigue Assessment Scale $)^{25}$; medications; and habitual dietary intake $(24 \mathrm{~h}$ recall). Physical activity (walking and standing) and sitting (sitting/lying) time were measured using the activPAL3 monitor (activPAL3; PAL Technologies Ltd, Glasgow, Scotland), positioned on the thigh of the nonparetic limb. The activPAL3 monitor recorded physical activity and sitting time for a minimum of 3 days prior to, during, and post experimental conditions. ProcessingPAL software (version 1.3; Leicester, United Kingdom), using validated algorithms, determined valid monitor wear time during waking hours. A day was deemed valid if (1) time spent in one activity was
$<95 \%$ of a day and (2) monitor wear time was $>10$ hours per day, ${ }^{26}$ and participants took $>100$ steps.

\section{Experimental Conditions}

In each cohort, participants completed an 8-hour control condition (prolonged uninterrupted sitting) and 2 experimental conditions (Tables 1 and 2), separated by a minimum 4-day washout period. Participants were provided with an individualized meal plan and a standardized evening meal 24 hours prior to commencing each condition. Individual energy requirements for prescribed meals were estimated from the Schofield equation ${ }^{27}$ for calculating basal metabolic rate, using weight, age, and appropriate physical activity levels $(1.3=$ sedentary adults). Participants were instructed 48 hours prior to conditions to abstain from exercise (moderateto vigorous-intensity physical activity), caffeine, and alcohol.

On arrival following a 12-hour overnight fast, an intravenous cannula was inserted into the upper limb and fasting blood samples were collected at 08:00 and 08:30. Participants consumed a standardized breakfast from 08:30 to 09:00. The trial began at 09:00 and ceased at 16:00. Under supervised conditions, participants raised from a seated position only to complete the prescribed 5-minute doses of STAND-EX (Table 2), or to void. Each STANDEX bout consisted of calf raises, mini squats, and marching on the spot (20 s on each exercise, repeated 5 times) completed at a Borg rating of perceived exertion of $\leq 3$ (light intensity). ${ }^{28}$ At 12:30 to 13:00, participants consumed a standardized lunch. Water intake (ad libitum) was recorded during the control condition and kept consistent throughout conditions.

Breakfast comprised of white bread (toasted) with a choice of butter, honey, or jam, prepacked cereal (Special K or corn flakes) with semiskimmed milk, apple, or orange juice and decaffeinated coffee or tea. Lunch comprised a frozen prepackaged meal (lean cuisine), a choice of apple or orange juice and a fruit cup ( 2 fruits or peach). Standardized meals were modified accordingly to contain approximately one-third of participants' estimated daily energy requirements, with a combined macronutrient content of $61 \%$ carbohydrate, $20 \%$ fat, and $16 \%$ protein.

\section{Outcome Measures and Blood Analysis}

The predetermined secondary outcome was within-participant, between-condition differences in postprandial plasma glucose response. Postprandial glucose is reported as the positive incremental area under the curve (+iAUC) calculated using the trapezoidal method. The +iAUC describes the positive area under the curve above the baseline value (08:00 AM fasting blood sample). ${ }^{29}$ Any value below baseline was treated as the 08:00 AM baseline value. Blood samples were collected in a 4-mL ethylenediaminetetraacetic acid tube every 30 minutes during each 2-hour postprandial period (Table 3), prior to scheduled bouts of STAND-EX. Blood samples were refrigerated immediately and were centrifuged (15 min at $-2000 g)$ on average within $18(0.01)$ minutes from collection. Plasma aliquots were stored at $-80^{\circ} \mathrm{C}$ for later analyses. All plasma glucose was analyzed in duplicate using the Abbott Point of Care blood analysis device (i-STAT; Abbott Point of Care Inc, Sydney, Australia).

\section{Data Analysis}

A statistical analysis plan was outlined a priori ${ }^{21}$ to detect withinperson, between-condition differences in the primary outcome systolic blood pressure. The secondary outcome postprandial 


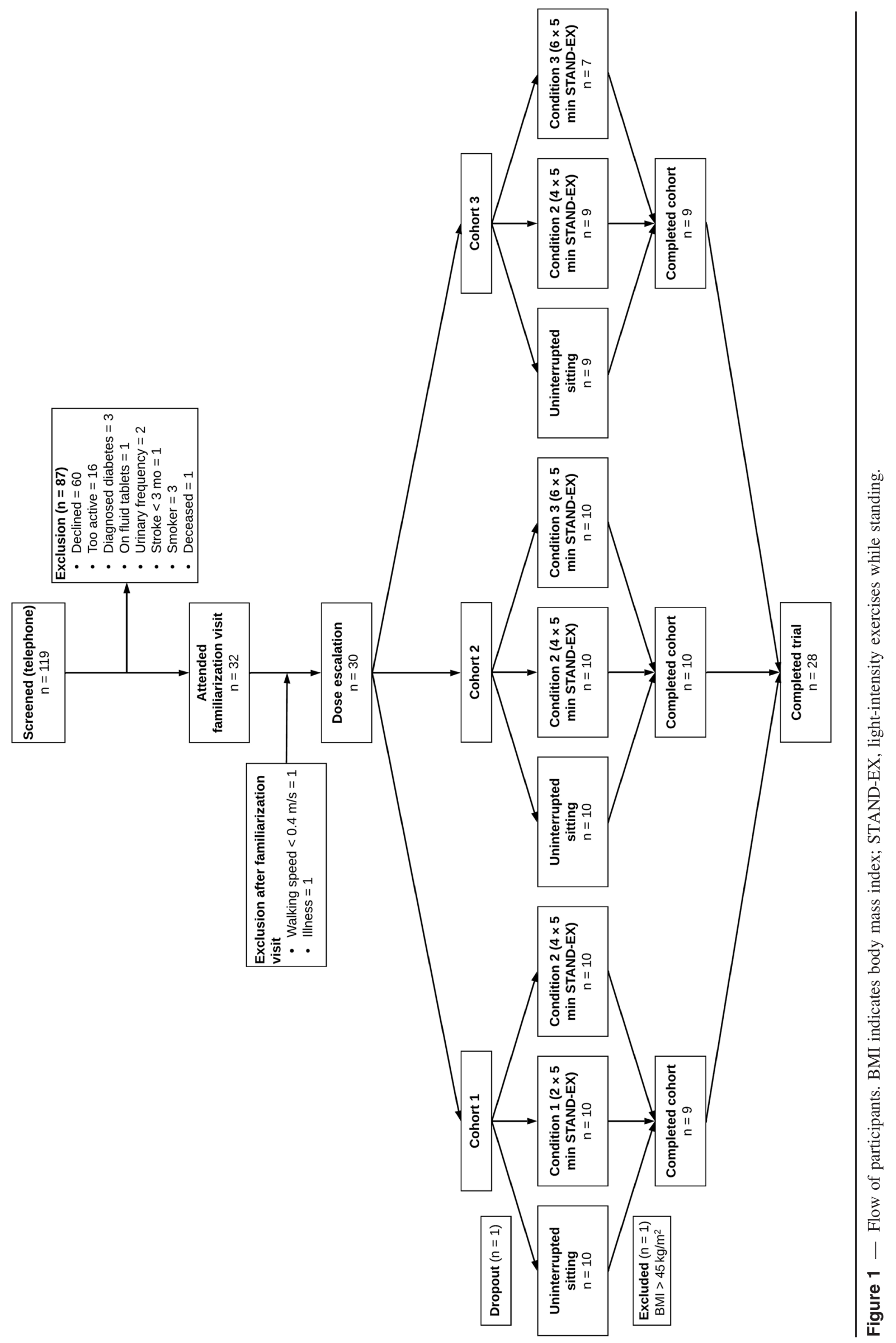


Table 1 Experimental Conditions by Cohort

\begin{tabular}{|c|c|c|c|c|c|c|}
\hline & $\begin{array}{c}\text { Sitting } \\
(8 \mathrm{~h})\end{array}$ & $\begin{array}{l}\text { Condition } 1 \\
(2 \times 5 \mathrm{~min})\end{array}$ & $\begin{array}{l}\text { Condition } 2 \\
(4 \times 5 \mathrm{~min})\end{array}$ & $\begin{array}{l}\text { Condition } 3 \\
(6 \times 5 \mathrm{~min})\end{array}$ & $\begin{array}{l}\text { Condition } 4 \\
(8 \times 5 \mathrm{~min})\end{array}$ & $\begin{array}{l}\text { Condition } 5 \\
(10 \times 5 \mathrm{~min})\end{array}$ \\
\hline \multicolumn{7}{|l|}{ Cohort 1} \\
\hline \multicolumn{7}{|l|}{ Cohort 2} \\
\hline \multicolumn{7}{|l|}{ Cohort 3} \\
\hline \multicolumn{7}{|l|}{ Cohort 4} \\
\hline Cohort 5 & & & & & & \\
\hline
\end{tabular}

glucose is reported as the positive incremental area under the curve (+iAUC). Simple imputation methods were used to impute a small proportion of missing values. Each missing value was imputed as a simple average of 2 values: (1) the mean of the 2 observed values at immediately adjacent timepoints within the participant, and (2) the mean observed value at the missing timepoint between participants. When a participant also had a missing value at either adjacent timepoint, the missing value was imputed simply as the value from (2).

Linear mixed models were used to estimate between-condition differences in glucose +iAUC, controlling for condition and time (fixed effects), and repeated measures on participants (using a random intercept). The initial morning meal has been suggested to contribute to daily variations in glycemic control. ${ }^{30}$ Fletcher et al ${ }^{30}$ reported in adolescents a significantly greater glucose incremental area under the curve (iAUC) following the breakfast only. In stroke survivors ${ }^{19}$ and those with type 2 diabetes, ${ }^{18}$ glucose concentrations were also demonstrated to be greater following the morning breakfast meal. Therefore, exploratory analysis examined between-condition effects in glucose +iAUC during the 3-hour morning period (08:0011:00). Statistical significance was set at $5 \%(P<.05)$ for all analyses. Data are represented as mean (SD) unless otherwise stated.

\section{Results}

\section{Participants}

Thirty-two participants were recruited and completed the familiarization session (Figure 1). Three withdrew and one was excluded due to not meeting the inclusion criteria (body mass index $>45 \mathrm{~kg} / \mathrm{m}^{2}$ ). Overall, 28 participants were included in the final analysis and 26 completed all experimental conditions (Figure 1). Due to the COVID-19 pandemic (2020), 2 participants were unable to complete their final visit $(6 \times 5$ min condition $)$ and the final participant could not be recruited. Three experimental conditions were tested $(2 \times 5 \mathrm{~min}, 4 \times 5 \mathrm{~min}$, and $6 \times 5 \mathrm{~min})$.

Table 3 details participant characteristics. Participants were on average 74 (90) months post stroke, with a mean age of 67 (13) years, and were classified as having a minor stroke (National Institute of Health Stroke Scale score 1-4). Participants spent on average 10.4 (2.9) hours per day sitting, 3.5 (1.7) hours per day standing, and 1.2 (0.6) hours per day walking over the (minimum) 4 days prior to completing their first experimental condition. On the days after the experimental conditions, participants spent slightly more time standing and walking, but the magnitude of this difference is not clinically important. For example, participants took on average 348 more steps ( $95 \%$ confidence interval [CI], 20 to 676) in the days following the $2 \times 5$ minutes condition, compared with the 4 days prior to their first experimental condition. Participants also spent 0.5 hours per day more in standing (95\% CI, 0.1 to 0.9 ) in the days following the $6 \times 5$ minutes condition compared with the 4 days prior to completing their first experimental condition.

\section{Effects of STAND-EX on Postprandial Glucose}

Table 4 details between-condition differences in glucose +iAUC during the 8-hour experimental conditions and the 3-hour morning period. Overall, 133 (12\%) data points were missing across all participants.

Glucose +iAUC was significantly decreased during the $4 \times 5$ minutes condition compared with prolonged sitting $(-1.1 \mathrm{mmol} / \mathrm{L}$. $7 \mathrm{~h} ; 95 \% \mathrm{CI},-2.0$ to -0.1 ) (Figure 2 ). Glucose +iAUC did not differ significantly in the $2 \times 5$ minutes condition or the $6 \times 5$ minutes condition, compared with prolonged sitting. During the 3 -hour morning period, glucose +iAUC was significantly lower during both the $4 \times 5$ minutes condition $(-0.8 \mathrm{mmol} / \mathrm{L} \cdot 3 \mathrm{~h} ; 95 \% \mathrm{CI},-1.3$ to $-0.3)$ and $6 \times 5$ minutes condition $(-0.8 \mathrm{mmol} / \mathrm{L} \cdot 3 \mathrm{~h} ; 95 \% \mathrm{CI},-1.5$ to -0.2 ) compared with prolonged sitting (Figure 2 ). The glucose + iAUC did not significantly differ in the $2 \times 5$ minutes condition compared with prolonged sitting.

\section{Discussion}

In stroke survivors, interrupting 8 hours of prolonged sitting during the $4 \times 5$ minutes condition (equating to one bout every $90 \mathrm{~min}$ ) with light-intensity standing-based exercises decreased the postprandial glucose response (15\% reduction) over 8 hours. A decrease in postprandial glucose was also found during the morning period (08:00-11:00) of the $4 \times 5$ minutes $(21 \%$ reduction) and $6 \times 5$ minutes $(17 \%$ reduction) conditions, when compared with prolonged sitting.

This trial builds on the evidence that has shown that frequently interrupting prolonged sitting time with bouts of physical activity or standing improves measures of cardiometabolic health ${ }^{16,31}$ and adds new insights into the morning postprandial glucose responses in stroke survivors. Elevations in 2-hour postprandial glucose are associated with an increased risk of mortality, cardiovascular disease, ${ }^{32,33}$ ischemic stroke, and coronary heart disease. ${ }^{34}$ Repeated glucose elevations have been shown to be associated with an increased risk of diabetes-related and cardiovascular complications, inducing an increase in oxidative stress, resulting in endothelial dysfunction. ${ }^{33,35}$ Our findings are consistent with other 


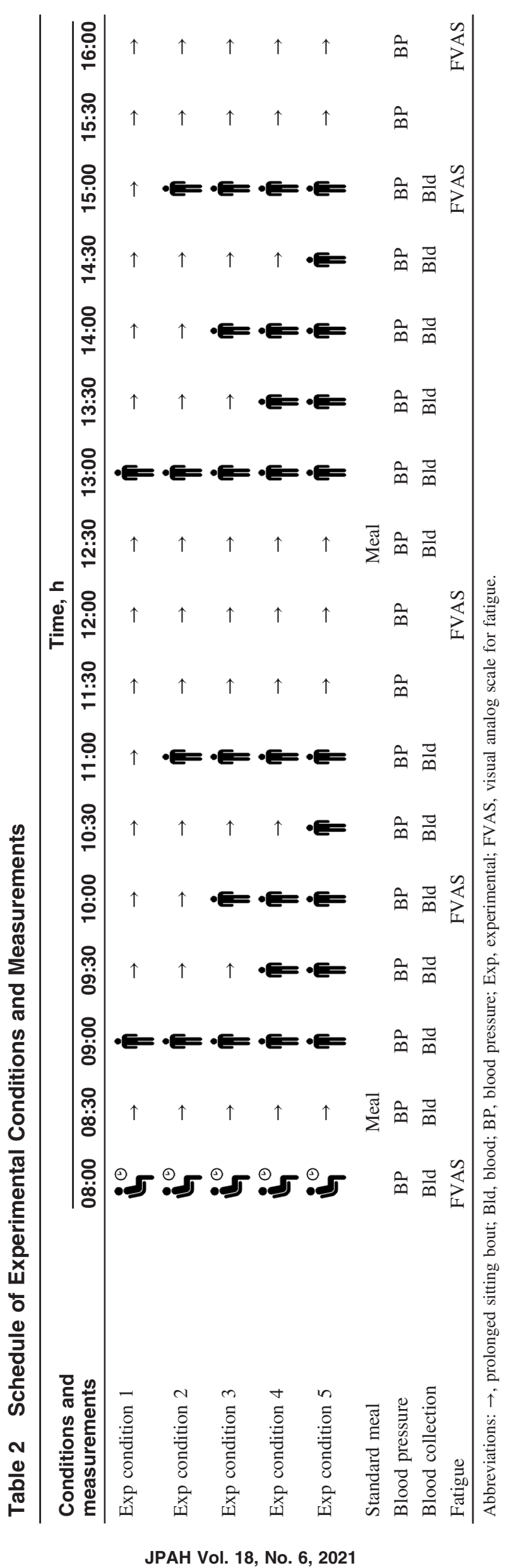


Table 3 Characteristics of Participants

\begin{tabular}{|c|c|c|c|c|}
\hline Characteristics & $\begin{array}{c}\text { Cohort } 1 \\
(n=9)\end{array}$ & $\begin{array}{c}\text { Cohort } 2 \\
(n=10)\end{array}$ & $\begin{array}{c}\text { Cohort } 3 \\
(n=9)\end{array}$ & $\begin{array}{c}\text { Total } \\
(\mathbf{N}=\mathbf{2 8})\end{array}$ \\
\hline Age, y (mean [SD]) & $66(15)$ & $67(8)$ & $67(16)$ & $67(13)$ \\
\hline Sex, number male $(\%)$ & $5(56)$ & $4(40)$ & $6(67)$ & $15(54)$ \\
\hline Weight, kg (mean [SD]) & $87.4(12.0)$ & $81.8(16.9)$ & $89.9(23.3)$ & $86(18)$ \\
\hline Height, m (mean [SD]) & $1.67(0.11)$ & $1.65(0.08)$ & $1.73(0.08)$ & $1.68(0.09)$ \\
\hline BMI, kg/m² (mean [SD]) & $31.3(2.7)$ & $30.2(6.1)$ & $29.9(5.9)$ & $30.4(5.0)$ \\
\hline Waist circumference, $\mathrm{cm}$ (mean [SD]) & $102(12)$ & $100(14)$ & $105(17)$ & $103(14)$ \\
\hline Time since stroke, mo (mean [SD]) & $96(105)$ & $46(52)$ & $81(108)$ & $74(90)$ \\
\hline AUSDRISK, mean (SD) & $14(5)$ & $17(4)$ & $17(5)$ & $16(5)$ \\
\hline \multicolumn{5}{|l|}{ Medications, number yes (\%) } \\
\hline Anticoagulants & $7(78)$ & $8(80)$ & $4(44)$ & $19(68)$ \\
\hline Antidepressants & $2(22)$ & $2(20)$ & $0(0)$ & $4(14)$ \\
\hline Cholesterol lowering & $4(44)$ & $7(70)$ & $5(56)$ & $16(57)$ \\
\hline Antihypertensive & $4(44)$ & $6(60)$ & $6(67)$ & $16(57)$ \\
\hline Walking aid, number yes (\%) & $7(78)$ & $8(80)$ & $5(56)$ & $7(25)$ \\
\hline Walking speed, m/s (mean [SD]) & $1.1(0.3)$ & $1.2(0.2)$ & $0.8(0.3)$ & $1.0(0.3)$ \\
\hline National Institute of Health Stroke Scale, mean (SD) & $3.0(3.9)$ & $2.3(2.2)$ & $4.6(4.9)$ & $3.2(3.7)$ \\
\hline No symptoms (score 0), number yes (\%) & $2(22)$ & $2(20)$ & $0(0)$ & $4(15)$ \\
\hline Mild (score 1-4), number yes (\%) & $5(56)$ & $6(60)$ & $5(63)$ & $16(60)$ \\
\hline Moderate (score 5-15), number yes (\%) & $2(22)$ & $2(20)$ & $3(38)$ & $7(27)$ \\
\hline \multicolumn{5}{|l|}{ Oxfordshire classification categories, number (\%) } \\
\hline TACI & $0(0)$ & $1(11)$ & $1(11)$ & $2(7)$ \\
\hline PACI & $3(33)$ & $4(44)$ & $4(44)$ & $11(41)$ \\
\hline Lacunar & $2(22)$ & $0(0)$ & $0(0)$ & $2(7)$ \\
\hline POCI & $1(11)$ & $0(0)$ & $0(0)$ & $1(4)$ \\
\hline Hemorrhage & $3(33)$ & $4(44)$ & $4(44)$ & $11(41)$ \\
\hline Side of hemiparesis, number left (\%) & $5(56)$ & $6(60)$ & $4(44)$ & $15(54)$ \\
\hline No hemiparesis, number yes (\%) & $0(0)$ & $1(10)$ & $0(0)$ & $1(4)$ \\
\hline
\end{tabular}

Abbreviations: BMI, body mass index; AUSDRISK, Australian type 2 diabetes risk; PACI, partial anterior circulation infarct; POCI, posterior circulation infarct; TACI, total anterior circulation infarct.

Table 4 Mean (SD) of Conditions for Glucose and +iAUC Across a Day and During the Morning, and Estimated Mean (95\% Cl) Difference Between Conditions

\begin{tabular}{|c|c|c|c|c|c|c|c|}
\hline \multirow[b]{2}{*}{ Outcome } & \multicolumn{4}{|c|}{ Conditions } & \multicolumn{3}{|c|}{ Difference between conditions } \\
\hline & $\begin{array}{c}\text { Sitting, } \\
n=28\end{array}$ & $\begin{array}{c}2 \times 5 \mathrm{~min} \\
\text { condition, } \\
n=9\end{array}$ & $\begin{array}{c}4 \times 5 \mathrm{~min} \\
\text { condition, } \\
n=28\end{array}$ & $\begin{array}{c}6 \times 5 \min \\
\text { condition, } \\
n=17\end{array}$ & $\begin{array}{c}2 \times 5 \text { min } \\
\text { condition } \\
\text { minus sitting }\end{array}$ & $\begin{array}{c}4 \times 5 \text { min } \\
\text { condition } \\
\text { minus sitting }\end{array}$ & $\begin{array}{c}6 \times 5 \text { min } \\
\text { condition } \\
\text { minus sitting }\end{array}$ \\
\hline Fasting glucose, $\mathrm{mmol} / \mathrm{L}$ & $5.8(0.9)$ & $5.4(0.3)$ & $5.8(1.1)$ & $6.0(1.2)$ & $0.05(-0.21$ to 0.31$)$ & $0.1(-0.1$ to 0.3$)$ & $-0.02(-0.22$ to 0.17$)$ \\
\hline $\begin{array}{l}\text { Average glucose across } \\
\text { day, } \mathrm{mmol} / \mathrm{L}\end{array}$ & $6.9(1.8)$ & $6.4(1.2)$ & $6.7(1.7)$ & $6.9(1.8)$ & $-0.1(-0.4$ to 0.2$)$ & $-0.1(-0.3$ to 0.1$)$ & $-0.2(-0.4$ to 0.1$)$ \\
\hline $\begin{array}{l}\text { Glucose }+ \text { iAUC, } \\
\mathrm{mmol} / \mathrm{L} \cdot 7 \mathrm{~h}\end{array}$ & $8.6(4.3)$ & $7.1(2.9)$ & $7.3(4.5)$ & $7.7(4.4)$ & $-0.4(-2.0$ to 1.1$)$ & $-1.1(-2.0$ to -0.1$)$ & $-0.9(-2.1$ to 0.3$)$ \\
\hline $\begin{array}{l}\text { Glucose }+\mathrm{iAUC} \\
\text { morning, } \mathrm{mmol} / \mathrm{L} \cdot 3 \mathrm{~h}\end{array}$ & $4.8(2.3)$ & $3.5(1.4)$ & $3.8(2.2)$ & $4.0(2.5)$ & $-0.5(-1.3$ to 0.4$)$ & $-0.8(-1.3$ to -0.3$)$ & $-0.8(-1.5$ to -0.1$)$ \\
\hline $\begin{array}{l}\text { Degree of difficulty, } \\
\text { RPE } 0-10 ; \\
0=\text { not difficult }\end{array}$ & $0.9(1.8)$ & $0.7(1.3)$ & $0.8(1.5)$ & $0.2(0.6)$ & $-0.4(-1.1$ to 0.4$)$ & $-0.2(-0.7$ to 0.3$)$ & $-0.4(-1.0$ to 0.2$)$ \\
\hline $\begin{array}{l}\text { Fatigue, VAS } 0-10 ; \\
0=\text { not fatigued }\end{array}$ & $2.1(2.1)$ & $2.1(2.4)$ & $1.7(2.3)$ & $1.0(1.5)$ & $-0.6(-1.2$ to -0.1$)$ & $-0.4(-0.7$ to -0.1$)$ & $-0.7(-1.1$ to -0.3$)$ \\
\hline
\end{tabular}

Abbreviations: +iAUC, positive incremental area under the curve; CI, confidence interval; RPE, ratings of perceived exertion; VAS, visual analog scale. 

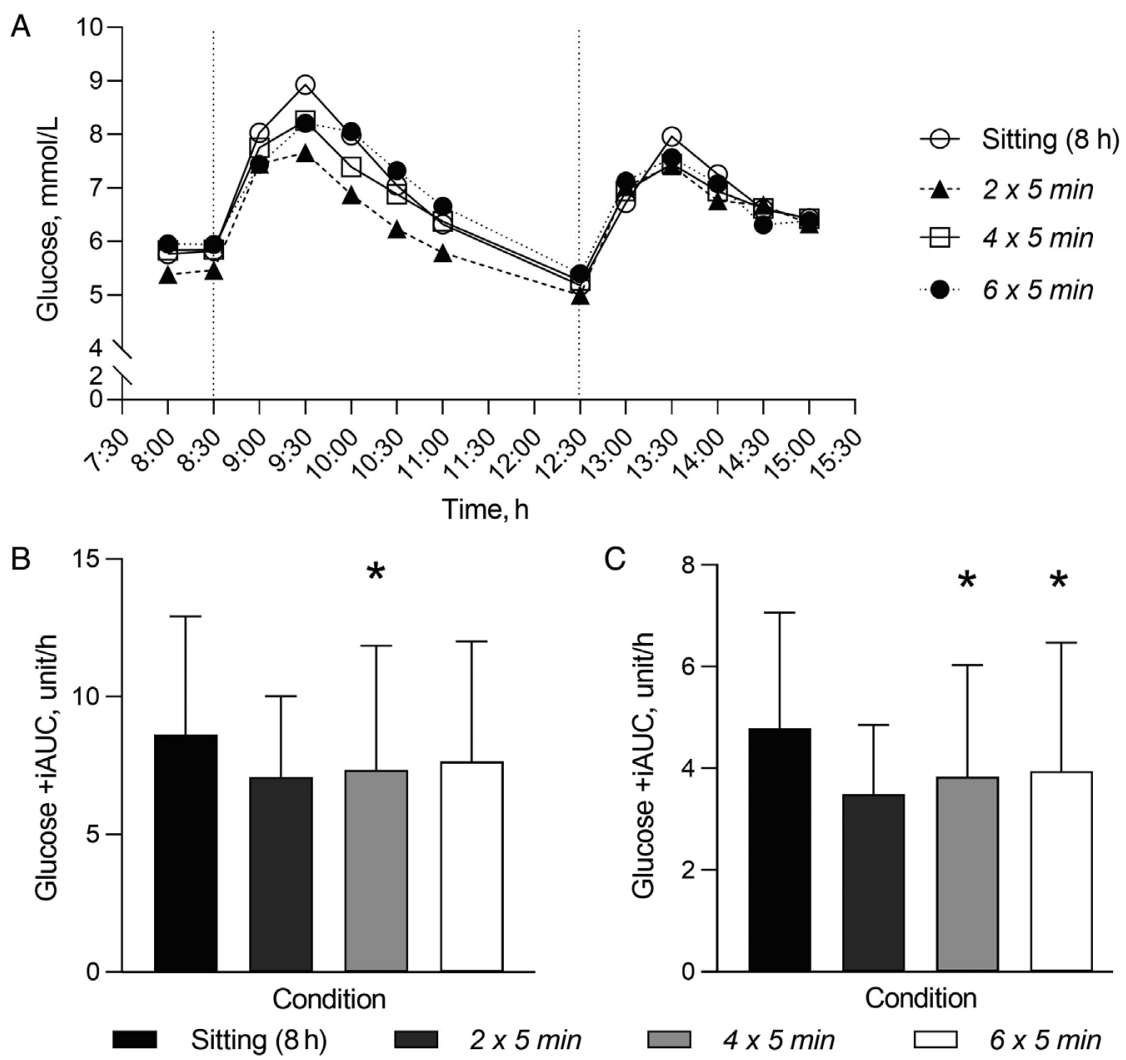

Figure 2 - (A) Mean glucose responses across a day during each condition. (B) and (C) Mean (SD) glucose + iAUC across a day and during the morning period (08:00-11:00), respectively. Vertical dashed lines in (A) indicate breakfast (08:30) and lunch (12:30) meals. +iAUC indicates positive incremental area under the curve. ${ }^{*} P<.05$.

clinical populations that report an acute decrease in postprandial glucose of between $24 \%$ and $39 \%$ in response to frequent short bouts of activity in those who are healthy, overweight or obese, or have type 2 diabetes. ${ }^{18,36,37}$ However, bouts of walking and simple resistance activities in these studies were more frequent (every 20 $30 \mathrm{~min}$ ) compared with the current trial (every 60-90 min). Taking into consideration that many stroke survivors are unable to meet the minimum recommended physical activity guidelines, ${ }^{38-40}$ our findings suggest that interrupting prolonged sitting every 60 to $90 \mathrm{~min}$ utes might be a feasible approach in stroke survivors with mild walking disability. Furthermore, interrupting prolonged sitting with frequent bouts of standing-based exercises might be an important strategy in controlling postprandial glucose regulation and consequently, could help to reduce the risk of developing cardiovascular and diabetes complications.

In contrast to our results, the only previous trial conducted in stroke survivors (BUST-Stroke trial) ${ }^{19}$ reported no significant differences in postprandial glucose when 8 hours of prolonged sitting was interrupted (every $30 \mathrm{~min}$ ) with 3 -minute bouts of STAND-EX or walking. ${ }^{19}$ The characteristics of included participants in BUST-Stroke were similar to our trial (mean age, 68 and $67 \mathrm{y}$; National Institute of Health Stroke Scale, 3.6 and 3.2; Australian type 2 diabetes risk, 16 and 16; body mass index 29.9 and 30.4, respectively). The discrepancy in results between BUST-Stroke and our study may be due to differences in methodology. In the BUST-Stroke trial, ${ }^{19}$ blood samples were processed within 1 to 2 hours after collection in comparison with the average processing time in our trial of $18(0.01)$ minutes. Delays of up to 2 hours in blood processing can lead to reductions of $46 \%$ in plasma glucose concentrations, compared within baseline concentrations. ${ }^{41}$ Thus, the longer and more varied time between blood collection and blood processing in the BUST-Stroke trial might partly explain the variations in postprandial glucose response. In addition, the high intraassay coefficient of variants reported in the BUST-Stroke trial $(<15 \%)$ might have also influenced results. Therefore, well-controlled blood processing designs are essential in future trials to confirm the effects of interrupting prolonged sitting time on postprandial glucose response in stroke survivors.

The primary strength of this trial was the timely processing of blood samples, and control of confounding variables. Although blood analysis was not blinded, blood samples were analyzed using the Abbott point of care analysis system (I-STAT) in duplicate. However, the following limitations must be considered. This trial was powered to detect within-person, between-condition differences in the primary outcome (systolic blood pressure). Therefore, we may have been underpowered to detect significant differences at the lower doses for postprandial glucose. In addition, exploratory analysis during the 3-hour morning period were based upon assumptions from previous trials ${ }^{18,19,30}$ and limits conclusions on experimental condition effects. Finally, no multiple adjustment testing was performed due to the small sample size of cohorts, increasing the risk of type I errors.

Engaging in light-intensity standing-based exercises every 90 minutes was observed to attenuate postprandial glucose during the morning, and over an 8-hour day in stroke survivors. More frequent (every $60 \mathrm{~min}$ ) bouts of standing-based exercises also attenuated postprandial glucose during the morning period. However, the magnitude of reduction did not differ between the 
$4 \times 5$ minutes and $6 \times 5$ minutes conditions during the morning period. These findings suggest the importance of frequently interrupting prolonged sitting for postprandial glucose control in stroke survivors. However, the effect of interrupting prolonged sitting time in glucose regulation in real-world settings and over longer periods, still needs to be assessed.

\section{Acknowledgments}

The authors thank all stroke survivors who participated in the trial. The authors also acknowledge the help of the Hunter Stroke Research Volunteer Register in participant recruitment and from Shaun Eslick (University of Newcastle) and Rebecca McLoughlin (University of Newcastle) who conducted the dietary assessments. This study is supported by a Hunter Medical Research Institute project grant (2016) and Heart Foundation Vanguard Grant (2017-no. 101727). P.M. (PhD candidate) is supported by a University of Newcastle PhD scholarship, and C.E. is supported by a Heart Foundation Future Leader Fellowship (no. 101177). D.D. is supported through an NHMRC Senior Research Fellowship (NHMRCAPP1078360) and the Victorian Government's Operational Infrastructure Support Program. H.J. is supported through a NSW Health EMC Clinical Research Fellowship (2017-2019) and the Hunter Medical Research Institute Women in Stroke Rehabilitation Research Fellowship (2019-2021). This trial was registered with the Australian New Zealand Clinical Trials Registry (ACTRN12617001517369). There were no competing interests to declare.

\section{References}

1. Bellettiere J, Winkler EAH, Chastin SFM, et al. Associations of sitting accumulation patterns with cardio-metabolic risk biomarkers in Australian adults. PLoS One. 2017;12(6):e0180119. PubMed ID: 28662164 doi:10.1371/journal.pone.0180119

2. Chastin SF, Egerton T, Leask C, Stamatakis E. Meta-analysis of the relationship between breaks in sedentary behavior and cardiometabolic health. Obesity. 2015;23(9):1800-1810. PubMed ID: 26308477 doi:10.1002/oby. 21180

3. Biswas A, Oh PI, Faulkner GE, et al. Sedentary time and its association with risk for disease incidence, mortality, and hospitalization in adults: a systematic review and meta-analysis. Ann Intern Med. 2015;162(2):123-132. PubMed ID: 25599350 doi:10.7326/ M14-1651

4. Homer AR, Owen N, Dunstan DW. Too much sitting and dysglycemia: mechanistic links and implications for obesity. Curr Opin Endocr Metab Res. 2019;4:42-49.

5. Lau L-H, Lew J, Borschmann K, Thijs V, Ekinci EI. Prevalence of diabetes and its effects on stroke outcomes: a meta-analysis and literature review. J Diabetes Investig. 2019;10(3):780-792. PubMed ID: 30220102 doi:10.1111/jdi.12932

6. Shou J, Zhou L, Zhu S, Zhang X. Diabetes is an independent risk factor for stroke recurrence in stroke patients: a meta-analysis. J Stroke Cerebrovasc Dis. 2015;24(9):1961-1968. PubMed ID: 26166420 doi:10.1016/j.jstrokecerebrovasdis.2015.04.004

7. Mapoure YN, Ba H, Ayeah CM, Kenmegne C, Luma HN, Njamnshi AK. Acute stroke patients with newly diagnosed diabetes mellitus have poorer outcomes than those with previously diagnosed diabetes mellitus. J Stroke Cerebrovasc Dis. 2018;27(9):2327-2335. PubMed ID: 29784608 doi:10.1016/j.jstrokecerebrovasdis.2018. 04.017

8. Fonville S, Zandbergen AAM, Koudstaal PJ, Den Hertog HM. Prediabetes in patients with stroke or transient ischemic attack: prevalence, risk and clinical management. Cerebrovasc Dis. 2014; 37(6):393-400. PubMed ID: 24993381 doi:10.1159/000360810

9. Zhang X, Shi Q, Zheng H, et al. Prevalence of abnormal glucose regulation according to different diagnostic criteria in ischaemic stroke without a history of diabetes. BioMed Res Int. 2018;2018: 8358724. PubMed ID: 29951547 doi:10.1155/2018/8358724

10. Matz K, Keresztes K, Tatschl C, et al. Disorders of glucose metabolism in acute stroke patients: an underrecognized problem. Diabetes Care. 2006;29(4):792-797. PubMed ID: 16567817 doi:10.2337/ diacare.29.04.06.dc05-1818

11. Hayes C, Kriska A. Role of physical activity in diabetes management and prevention. J Am Diet Assoc. 2008;108(4)(suppl 1):S19-S23. PubMed ID: 18358249 doi:10.1016/j.jada.2008.01.016

12. Boysen G, Krarup L-H. Benefits of physical activity for stroke survivors. Exp Rev Neurother. 2009;9(2):147-149. PubMed ID: 19210189 doi:10.1586/14737175.9.2.147

13. Hendrickx W, Riveros C, Askim T, et al. Identifying factors associated with sedentary time after stroke. Secondary analysis of pooled data from nine primary studies. Top Stroke Rehabil. 2019;26(5):327334. PubMed ID: 31025908 doi:10.1080/10749357.2019.1601419

14. English C, Healy GN, Coates A, Lewis L, Olds T, Bernhardt J. Sitting and activity time in people with stroke. Phys Ther. 2016; 96(2):193-201. PubMed ID: 26112254 doi:10.2522/ptj.20140522

15. Butler EN, Evenson KR. Prevalence of physical activity and sedentary behavior among stroke survivors in the United States. Top Stroke Rehabil. 2014;21(3):246-255. PubMed ID: 24985392 doi:10.1310/ tsr2103-246

16. Mackie P, Weerasekara I, Crowfoot G, et al. What is the effect of interrupting prolonged sitting with frequent bouts of physical activity or standing on first or recurrent stroke risk factors? A scoping review. PLoS One. 2019;14(6):e0217981. PubMed ID: 31194799 doi:10. 1371/journal.pone.0217981

17. Saunders TJ, Atkinson HF, Burr J, Macewen B, Skeaff CM, Peddie MC. The acute metabolic and vascular impact of interrupting prolonged sitting: a systematic review and meta-analysis. Sports Med. 2018;48(10):2347-2366. PubMed ID: 30078066 doi:10.1007/s40279018-0963-8

18. Dempsey PC, Larsen RN, Sethi P, et al. Benefits for type 2 diabetes of interrupting prolonged sitting with brief bouts of light walking or simple resistance activities. Diabetes Care. 2016;39(6):964-972. PubMed ID: 27208318 doi:10.2337/dc15-2336

19. English C, Janssen H, Crowfoot G, et al. Breaking up sitting time after stroke (BUST-stroke). J Stroke. 2018;13(9):921-931. PubMed ID: 30226448 doi:10.1177/1747493018801222

20. English C, Janssen H, Crowfoot G, et al. Frequent, short bouts of light-intensity exercises while standing decreases systolic blood pressure: breaking up sitting time after stroke (BUST-Stroke) trial. J Stroke. 2018;13(9):932-940. PubMed ID: 30223728 doi:10.1177/ 1747493018798535

21. Mackie $P$, Crowfoot G, Janssen H, et al. Breaking up sitting time after stroke-how much less sitting is needed to improve blood pressure after stroke (BUST-BP-dose): protocol for a dose-finding study. Contemp Clin Trials Commun. 2018;13:100310. PubMed ID: 30740549 doi:10.1016/j.conctc.2018.100310

22. Kasner SE, Cucchiara BL, McGarvey ML, Luciano JM, Liebeskind DS, Chalela JA. Modified National Institutes of Health Stroke Scale can be estimated from medical records. Stroke. 2003;34(2):568-570. PubMed ID: 12574577 doi:10.1161/01.STR.0000052630.11159.25

23. Bamford J, Sandercock P, Dennis M, Warlow C, Burn J. Classification and natural history of clinically identifiable subtypes of cerebral infarction. Lancet. 1991;337(8756):1521-1526. PubMed ID: 1675378 doi:10.1016/0140-6736(91)93206-o 
24. Australian Government Department of Health. Australian type 2 diabetes risk assessment tool (AUSDRISK). 2016. https://www1. health.gov.au/internet/main/publishing.nsf/Content/chronic-diab-prevaus/\$File/austool5.pdf. Accessed May 2020.

25. Smith ORF, Van Den Broek KC, Renkens M, Denollet J. Comparison of fatigue levels in patients with stroke and patients with end-stage heart failure: application of the fatigue assessment scale. $J \mathrm{Am}$ Geriatr. 2008;56(10):1915-1919. PubMed ID: 18771452 doi:10. 1111/j.1532-5415.2008.01925.x

26. Winkler EAH, Bodicoat DH, Healy GN, et al. Identifying adults' valid waking wear time by automated estimation in activPAL data collected with a 24 h wear protocol. Physiol Meas. 2016;37(10): 1653-1668. PubMed ID: 27652827 doi:10.1088/0967-3334/37/10/ 1653

27. Schofield W. Predicting basal metabolic rate, new standards and review of previous work. Hum Nutr Clin Nutr. 1985;39(suppl 1): 5-41. PubMed ID: 4044297

28. Borg GA. Psychophysical bases of perceived exertion. Med Sci Sports Exerc. 1982;14(5):377-381. PubMed ID: 7154893

29. Brouns F, Bjorck I, Frayn K, et al. Glycaemic index methodology. Nutr Res Rev. 2005;18(1):145-171. PubMed ID: 19079901 doi:10. 1079/NRR2005100

30. Fletcher EA, Salmon J, McNaughton SA, et al. Effects of breaking up sitting on adolescents' postprandial glucose after consuming meals varying in energy: a cross-over randomised trial. J Sci Med Sport. 2018;21(3):280-285. PubMed ID: 28625540 doi:10.1016/j.jsams. 2017.06.002

31. Chastin SFM, De Craemer M, De Cocker K, et al. How does lightintensity physical activity associate with adult cardiometabolic health and mortality? Systematic review with meta-analysis of experimental and observational studies. Br J Sports Med. 2019;53(6):370-376. PubMed ID: 29695511 doi:10.1136/bjsports-2017-097563

32. Triplitt C, Alvarez CA. Best practices for lowering the risk of cardiovascular disease in diabetes. Diabetes. 2008;21(3):177-189.
33. Hatamoto Y, Goya R, Yamada Y, et al. Effect of exercise timing on elevated postprandial glucose levels. J Appl Physiol. 2017;123(2):278284. PubMed ID: 28408695 doi:10.1152/japplphysiol.00608.2016

34. Ning F, Zhang L, Dekker JM, et al. Development of coronary heart disease and ischemic stroke in relation to fasting and 2-hour plasma glucose levels in the normal range. Cardiovasc Diabetol. 2012; 11(1):76. PubMed ID: 22731255 doi:10.1186/1475-2840-11-76

35. Ceriello A. Postprandial glucose regulation and diabetic complications. Arch Intern Med. 2004;164(19):2090-2095. PubMed ID: 15505121 doi:10.1001/archinte.164.19.2090

36. Dunstan DW, Kingwell BA, Larsen R, et al. Breaking up prolonged sitting reduces postprandial glucose and insulin responses. Diabetes Care. 2012;35(5):976-983. PubMed ID: 22374636 doi:10.2337/ dc11-1931

37. Peddie MC, Bone JL, Rehrer NJ, Skeaff CM, Gray AR, Perry TL. Breaking prolonged sitting reduces postprandial glycemia in healthy, normal-weight adults: a randomized crossover trial. Am J Clin Nutr. 2013;98(2):358-366. PubMed ID: 23803893 doi:10.3945/ajcn.112. 051763

38. Fini NA, Holland AE, Keating J, Simek J, Bernhardt J. How physically active are people following stroke? Systematic review and quantitative synthesis. Phys Ther. 2017;97(7):707-717. PubMed ID: 28444348 doi:10.1093/ptj/pzx038

39. Field MJ, Gebruers N, Shanmuga Sundaram T, Nicholson S, Mead G. Physical activity after stroke: a systematic review and meta-analysis. ISRN Stroke. 2013;2013:464176. doi:10.1155/2013/464176

40. Billinger SA, Arena R, Bernhardt J, et al. Physical activity and exercise recommendations for stroke survivors: a statement for healthcare professionals from the American Heart Association/American Stroke Association. Stroke. 2014;45(8):2532-2553. PubMed ID: 24846875 doi:10.1161/STR.0000000000000022

41. Al salhen $\mathrm{K}$. The effect of storage time and different anticoagulants on fasting blood glucose concentration. Al-Mukhtar J Sci. 2018; 33(2):100-106. 